\title{
Effect of finite on cyclotron-wave propagation in metals in the long-wavelength limit
}

\author{
Frandsen, J. B.; Gordon, R A
}

Published in:

Physical Review B

Link to article, DOI:

10.1103/PhysRevB.14.4342

Publication date:

1976

Document Version

Publisher's PDF, also known as Version of record

Link back to DTU Orbit

Citation (APA):

Frandsen, J. B., \& Gordon, R. A. (1976). Effect of finite on cyclotron-wave propagation in metals in the longwavelength limit. Physical Review B, 14(10), 4342-4344. https://doi.org/10.1103/PhysRevB.14.4342

\section{General rights}

Copyright and moral rights for the publications made accessible in the public portal are retained by the authors and/or other copyright owners and it is a condition of accessing publications that users recognise and abide by the legal requirements associated with these rights.

- Users may download and print one copy of any publication from the public portal for the purpose of private study or research.

- You may not further distribute the material or use it for any profit-making activity or commercial gain

- You may freely distribute the URL identifying the publication in the public portal

If you believe that this document breaches copyright please contact us providing details, and we will remove access to the work immediately and investigate your claim. 


\title{
Effect of finite $\omega \tau$ on cyclotron-wave propagation in metals in the long-wavelength limit
}

\author{
J. B. Frandsen* and R. A. Gordon \\ Physics Laboratory I, The Technical University of Denmark, DK-2800 Lyngby, Denmark
}

(Received 26 January 1976)

\begin{abstract}
Exact calculations of cyclotron-wave dispersion curves have been carried out for a degenerate free-electron metal including finite relaxation time effects. The calculated dispersion curves are shown to differ substantially from the experimental dispersion curves in the long-wavelength limit. The nature of this disagreement is discussed in some detail and it is pointed out that finite relaxation time effects could lead to significant changes in the experimentally determined Fermi-liquid parameters $\left\{A_{i}\right\}$ for $i \geq 2$.
\end{abstract}

It is well known that so-called cyclotron waves can propagate on the high-field side of the AzbelKaner cyclotron resonance fundamental and its higher harmonics. ${ }^{1,2}$ Such waves are a direct manifestation of the long -wavelength collective response of a metal to an external electromagnetic field. Up until now, however, the interpretation of experimental curves has generally been based on a comparison with theoretical calculations which are rigorously valid only in the infinite $\omega \tau$ limit. $^{2,3}$ Here, $\omega$ is the angular frequency and $\tau$ is an "effective" relaxation time between electron collisions. It has been shown, however, that finite values of $\omega \tau$ significantly alter the theoretical dispersion curves in the long-wavelength limit for both the extraordinary ${ }^{4}$ as well as the ordinary ${ }^{5}$ cyclotron-wave polarizations.

It is the purpose of this communication to show quantitatively that the effect of a finite value of $\omega \tau$ must be taken into account in the interpretation of experimental measurements in the long-wavelength limit. To this end we present the results of numerical calculations for a degenerate Fermi gas of noninteracting electrons with a spherical Fermi surface where the effects of finite $\omega \tau$ can be taken into account exactly without any complications from band structure or other Fermi-surface parameters. We then briefly consider what modifications the addition of Fermi-liquid effects might have on the results and indicate that additional theoretical work will be required to provide an unambiguous interpretation of the cyclotronwave measurements in the long-wavelength limit.

The infinite-medium dispersion relation for cyclotron waves in a noninteracting electron Fermi gas follows immediately from Maxwell's equations

$$
\begin{aligned}
& q^{2}-i \omega \mu_{0} \sigma_{x x}=0, \\
& q^{2}-i \omega \mu_{0}\left(\sigma_{y y}+\sigma_{y z}^{2} / \sigma_{z z}\right)=0,
\end{aligned}
$$

where Eq. (1a) corresponds to the so-called ordinary polarization $(\overrightarrow{\mathrm{B}} \| \overrightarrow{\mathrm{E}})$ and Eq. (1b) corresponds to the so-called extraordinary polarization $(\vec{B} \perp \vec{E})$. Here, $\vec{E}$ is the high-frequency electric field, $\vec{B}$ is the dc magnetic field taken to lie along the $x$ axis, $\overrightarrow{\mathrm{q}}$ is the wave vector taken to lie along the $z$ axis, and $\mu_{0}$ is the magnetic permeability. It should be noted that the wave vector $\vec{q}$ is a complex quantity and that the angular frequency $\omega$ is taken as real. The components of the conductivity tensor $\sigma_{i j}$ have been given by Chambers. ${ }^{6}$ The numerical computations necessary to calculate $\sigma_{i j}$ exactly were greatly simplified by rewriting Chamber's expression as a single integral. Thus, $\sigma_{x x}$ for the ordinary polarization was calculated using

$$
\sigma_{x x}=\frac{3 i \epsilon_{0}}{\sin \pi \eta} \frac{\omega_{p}^{2}}{\omega_{c}} \int_{0}^{\pi} d \nu \cos \eta(\pi-\nu) \frac{F(K)}{K}
$$

where

$$
\eta \equiv\left(\omega / \omega_{c}\right)(1+i / \omega \tau)
$$

and

$$
F(K) \equiv F\left(q D \sin \frac{1}{2} \nu\right)=\cos K / K-\sin K / K^{2} .
$$

Here, $D$ is the cyclotron-orbit diameter and $\omega_{p}$ and $\omega_{c}$ are the plasma and cyclotron frequencies, respectively. A similar expression for the extraordinary polarization has been given before. ${ }^{7}$

Typical dispersion and attenuation curves calculated in such a manner are shown in Figs. 1 and 2 using the known values of the effective mass $m^{*}$ and Fermi radius $k_{F}$ for potassium. ${ }^{8}$ Here, $R_{0}$ is defined as $v_{F} / \omega$ where $v_{F}$ is the Fermi velocity. The exact experimental value of $\omega \tau$ is, of course, not precisely known but experimental values of $\omega \tau \sim 20-50$ are typical. ${ }^{9}$ The experimental points can be seen to be in reasonabiy good agreement with the theoretical curves for the "linear" portion of the dispersion curve but deviate significant ly in the long-wavelength limit. This long-wavelength deviation is shown more clearly in Fig. 3 for the so-called ordinary cyclotron waves above the cyclotron resonance fundamental. It is im portant to note the change in the shape of the dis persion curve and its shift towards smaller field values, i.e., away from the experimental points. These features are repeated for the extraordinary 


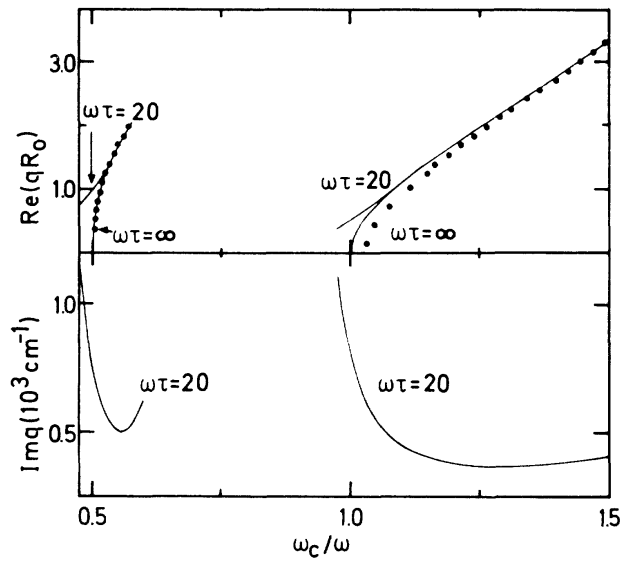

FIG. 1. Theoretical-dispersion $\operatorname{Re}\left(q R_{0}\right)$ and attenuation $\operatorname{Im}(q)$ curves for ordinary cyclotron waves at $17.35 \mathrm{GHz}$ in potassium in the free-electron limit. The circles denote experimentally determined points at $17.3 \mathrm{GHz}$ (see Ref. 16).

polarization and are equally well pronounced for the higher harmonics of both the ordinary and extraordinary polarizations.

We would like to emphasize that these deviations can not result from uncertainties in the parameters used to calculate the theoretical dispersion curves. For example, it has been shown that the anisotropy in the Fermi surface of potassium is too small to lead to any significant change in the calculated dispersion curves. ${ }^{10,11}$ Similarly, we

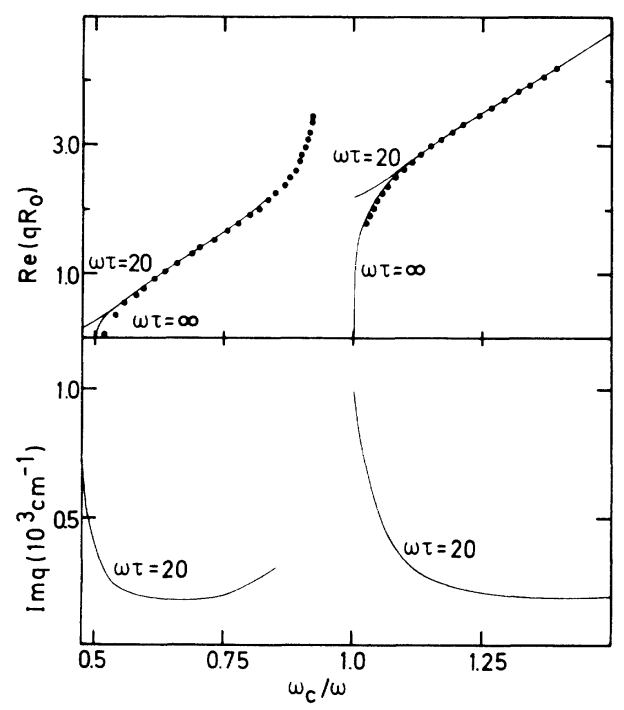

FIG. 2. Theoretical-dispersion $\operatorname{Re}\left(q R_{0}\right)$ and attenuation $\operatorname{Im}(q)$ curves for extraordinary cyclotron waves at $\mathbf{1 7 . 3 5}$ $\mathrm{GHz}$ in potassium in the free-electron limit. The circles denote experimentally determined points at $17.4 \mathrm{GHz}$ (see Ref. 3). have verified that the uncertainties or anisotropies in the Fermi radius or in the "long-wavelength" effective mass produce completely negligible changes in the dispersion curves in the long-wavelength limit. ${ }^{12}$ Likewise, the neglect of the slight momentum dependence of the relaxation time will not significantly affect the calculated dispersion curves - especially at low temperatures where such effects are negligible in comparison with the experimental uncertainty in $\tau .^{6,13,14}$ Thus it must be concluded that rigorous exact calculations including finite relaxation time effects for a Fermi gas can not account for the experimental cyclotron-wave dispersion curves for potassium in the long-wavelength limit.

We now briefly discuss what effect the introduction of Fermi-liquid effects might have on the calculations. The calculation of cyclotron-wave dispersion curves for a Fermi liquid is, of course, much more complicated than for a Fermi gas and exact detailed calculations taking into account both Fermi liquid and finite relaxation time effects have not been made. In the absence of such calculations we merely note the well-established result that the dominant effect of the Fermi-liquid interaction in the long -wavelength limit - for infinite $\omega \tau$-will be to shift the infinite $\omega \tau$ dispersion curve for a Fermi gas in magnetic field. ${ }^{3,15,16}$ If, however, such a displacement is carried out for a dispersion curve calculated for a Fermi gas with a realistic value of $\omega \tau$, it is found that a substantial disagreement with the experimental dispersion

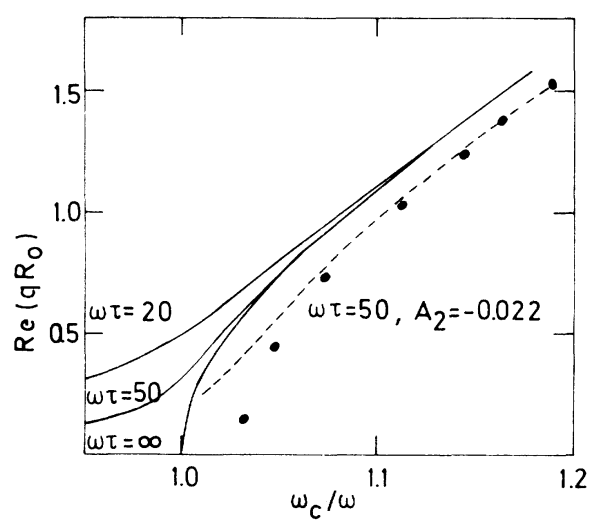

FIG. 3. Theoretical-dispersion $\operatorname{Re}\left(q R_{0}\right)$ curves for ordinary cyclotron waves near the cyclotron resonance fundamental at $17.35 \mathrm{GHz}$ in potassium in the free-electron limit. The circles denote experimentally determined points at $17.3 \mathrm{GHz}$ (see Ref. 16). The dotted line represents a displaced free-electron dispersion curve for $\omega \tau=50$ obtained by substituting $\left(\omega_{c} / \omega\right)+A_{2}$ for $\omega_{c} / \omega$ in the theoretical calculations. The value of $A_{2}\left(A_{2}\right.$ $=-0.022$ ) is taken to be equal to the relevant quoted experimental Fermi-liquid coefficient (see Ref. 12). 
curves still remains in the long-wavelength limit. This is illustrated in Fig. 3 where the $\omega \tau=50$ curve for a Fermi gas is displaced by an amount consistent with the experimentally determined Fermi-liquid coefficient, $A_{2} \cdot{ }^{12}$

Such an analysis of Fermi-liquid effects should, of course, not be regarded as rigorous. What is relevant to our discussion here, however, is that the rigorous inclusion of Fermi-liquid effects, in the long -wavelength limit, would not be expected to completely suppress the relatively large (even for $\omega \tau \gtrsim 50$ ) long-wavelength "tail" of the dispersion curve or its shift to lower-field values result ing from finite relaxation time effects in a Fermi gas. ${ }^{17}$ Accordingly, one must conclude that further theoretical work will be necessary to obtain an unambiguous interpretation of cyclotron-wave measurements in the long-wavelength limit. In partic- ular, the boundary-value problem for the power absorbed in finite slabs, due to the double-sided excitation of cyclotron waves for finite $\omega \tau$, will have to be definitively solved since the published experimental dispersion curves discussed here have been based on measurements of the absorbed power. Such calculations would be especially important since even slight changes in the experimentally-determined Fermi-liquid dispersion curve due to finite relaxation time effects could readily lead to significant changes in the quoted experimental values of the spin-independent Fermi-liquid coefficients $\left\{A_{i}\right\}$ for $i \geq 2$.

The authors would like to thank J. J. Quinn and S. C. Ying for a number of discussions of Fermi liquid effects and W. M. Walsh, Jr. for several discussions of the experimental dispersion curves.
* Present address: Dept. of Physics, University of Roorkee, Roorkee, India.

${ }^{1}$ E. A. Kaner and V. G. Skobov, Plasma Effects in Metals (Taylor and Francis, London, 1971), Chap. 7.

${ }^{2}$ W. M. Walsh, Jr. and P. M. Platzman, Phys. Rev. Lett. 15, 784 (1965).

${ }^{3}$ P. M. Platzman and P. A. Wolff, Waves and Interactions in Solid State Plasmas (Academic, London, 1973), Chaps. 8 and 10 .

${ }^{4} \mathrm{~J} . \mathrm{B}$. Frandsen, in Proceedings of the Fourteenth International Conference on Low Temperature Physics, edited by M. Krusius and M. Vuorio (North-Holland, Amsterdam, 1975), Vol. 4, p. 321.

${ }^{5}$ D. R. Fredkin and A. R. Wilson, in Proceedings of the Eleventh International Conference on Low Temperature Physics, edited by J. F. Allen, J. F. Finlayson, and D. M. McCall (University of St. Andrews, St. Andrews, Scotland, 1968), Vol. 2, p. 1178.

${ }^{6} \mathrm{R}$. G. Chambers, in The Physics of Metals, edited by J. M. Ziman (Cambridge U. P., Cambridge, England, 1969), p. 175 .
${ }^{7}$ J. B. Frandsen, L. E. Hasselberg, J. Lebech, and K. Saermark, Phys. Status Solidi B 67, 501 (1975).

${ }^{8}$ G. L. Dunifer, D. Pinkel, and S. Shultz, Phys. Rev. B 10, 3159 (1974).

${ }^{9}$ For typical experimental frequencies between 12 and $35 \mathrm{GHz}$, see W. M. Walsh, Jr. (private communication).

${ }^{10} \mathrm{~S}$. Matsuo, T. Yamada, and M. Tsuji, J. Phys. Soc. Jpn. 34, 897 (1973).

${ }^{11}$ D. R. Fredkin and R. Freeman, Phys. Rev. B $\underline{9}, 360$ (1974).

${ }^{12}$ W. M. Walsh, Jr., L. W. Rupp, Jr., P. H. Schmidt, and R. N. Castellano, Bull. Am. Phys. Soc. 18, 336 (1973).

${ }^{13}$ A. B. Pippard, Proc. R. Soc. A 305, 291 (1968).

${ }^{14}$ D. K. Wagner, R. C. Alberts, and M. Roy, Solid State Commun 15, 1337 (1974).

${ }^{15}$ S. C. Ying and J. J. Quinn, Phys. Rev. 180, 193 (1969).

${ }^{16}$ P. M. Platzman, W. M. Walsh, Jr., and E-Ni Foo, Phys. Rev. 172, 689 (1968).

${ }^{17}$ J. J. Quinn and S. C. Ying (private communication). 